\title{
Synthetic Lethality Induced by Toxic Polyglutamine Tract II: A Survey in Drosophila
}

\author{
Ping Zhang*, Daniel Camacho, Shashank Vodapally, Shanado Williams, Kavitha Kannan \\ Department of Molecular and Cell Biology, University of Connecticut, Storrs, USA \\ Email: *ping.zhang@uconn.edu
}

Received 19 March 2015; accepted 26 June 2015; published 29 June 2015

Copyright (C) 2015 by authors and Scientific Research Publishing Inc.

This work is licensed under the Creative Commons Attribution International License (CC BY).

http://creativecommons.org/licenses/by/4.0/

(c) (i) Open Access

\section{Abstract}

Mutant proteins containing an expanded polyglutamine tract induce cell death and cause neurodegenerative diseases. These toxic proteins interfere with a variety of physiological pathways, but the key interactions between the toxins and cellular factors remain unclear. To model the diseases in Drosophila, the GMR-Gal4/UAS gene expression system has been used extensively, which operates in the eyes. By using the system, genome-wide studies have resulted in the isolation of functionally diverse groups of Drosophila genes that interact with the disease proteins. We previously reported that coexpressing the Drosophila Dikar gene and an expanded polyglutamine tract by GMR-Gal4/UAS induced a synthetic lethality. We carried out follow-up experiments to isolate additional synthetic lethal alleles. Our data provide evidence that synthetic lethality associated with expressing an expanded polyglutamine tract is more common than thought to be and could have escaped the conventional genetic screens. Our results also suggest that 1) the gene expression system is leaky, allowing expression outside of the primary target eye cell types; 2) expressing an expanded polyglutamine tract is extremely toxic to cells; and 3) combining the leaky expression and the toxicity results in a lethal-prone condition. Thus, genetic modifications to the disease proteins' acute toxicity could frequently lead to synthetic lethality. However, synthetic lethal alleles are excluded from most conventional screens, necessitating alternative approaches such as a twostep method used in this study to isolate the modifiers. Since synthetic lethality reflects essential genetic buffering networks, studying these alleles may hold the keys to identify the critical interactions in the disease development between the toxic proteins and the physiological pathways.

\section{Keywords}

Polyglutamine Diseases, Drosophila, Genetic Screen, GMR-Gal4/UAS System, Synthetic Lethal,

${ }^{*}$ Corresponding author.

How to cite this paper: Zhang, P., Camacho, D., Vodapally, S., Williams, S. and Kannan, K. (2015) Synthetic Lethality Induced by Toxic Polyglutamine Tract II: A Survey in Drosophila. Open Journal of Genetics, 5, 58-70.

http://dx.doi.org/10.4236/ojgen.2015.52005 


\section{Mutations}

\section{Introduction}

When a protein contains a long tract of 40 or more glutamine amino acid residues, it acts as a cellular toxin, frequently resulting in cell death. In humans, a group of nine genes give rise to neurodegenerative diseases, when each is mutated to produce a toxic expanded polyglutamine tract [1]. While the fundamental questions of how an expanded polyglutamine tract leads to cellular toxicity remain unclear, studies using various genetic manipulations in model organisms have implicated an array of physiological pathways that influence or modify the detrimental effects of the expanded polyglutamine proteins [2]-[5]. For example, numerous cellular pathways have been implicated to influence Huntington's disease, including many aspects of mitochondrial functions [6], and transcriptional regulation [7]. In addition to the intrinsic toxicity of an expanded polyglutamine tract [8]-[10], studies have also shown that domains flanking the toxic polyglutamine tract in a disease protein contribute to the neurodegenerative disease development [11]-[13].

Using model organisms, large collections of modifiers have been isolated primarily by monitoring the effects of expressing expanded polyglutamine proteins in mutant backgrounds [14]-[16]. In Drosophila, the GMRGal4/UAS binary gene expression system, which operates in the eyes, is introduced as a tool to express the expanded polyglutamine proteins and to identify the modifiers [17] [18]. GMR-Gal4/UAS is based on a construct of GMR (glass multiple reporter) that carries multiple copies of an eye enhancer element to generate a conditional expression in the eyes [19] [20]. In genome-wide screens to identify modifiers, this system relied on modifications to the adult eye morphological characteristics. It is not only sensitive but also efficient in identifying the modifiers and has resulted in the identification of large groups of functionally diverse modifiers [17] [21].

Recently, we came across a synthetic lethality phenomenon resulting from coexpressing a toxic polyglutamine tract and a Drosophila gene, Dikar, with the GMR-Gal4/UAS system [22]. The synthetic lethality occurred when the simultaneous overexpression of two gene products, Dikar and an expanded polyglutamine tract, led to organismal death, whereas expressing either of them individually did not affect their viability. Since this was the first known case of synthetic lethality associated with expressing a toxic polyglutamine tract, we set out to explore the possibility that the neurotoxin-induced synthetic lethality might be a common phenomenon that has so far escaped detection. Here we describe the results from an effort to identify additional Drosophila alleles of this type of synthetic lethality. Our data suggest that the presence of a toxic polyglutamine tract induces a sensitized lethal-prone condition that could easily lead to synthetic lethality when combined with mutations.

\section{Materials and Methods}

\subsection{Drosophila Strains and Culture}

Flies were cultured on standard corn/agar media at $25^{\circ} \mathrm{C}$, unless otherwise stated. The strains and alleles used in this study were obtained from the Bloomington Drosophila Stock Center and other sources as described previously [22]. Through meiotic recombination, we generated a set of stocks, each of which contains a Chromosome 2 carrying two transgenes. These include 1) longGMR-Gal4>UAS-httex1p-97QP-s/CyO, 2) longGMRGal4>UAS-httex1p-97QP-w/CyO, and 3) longGMR-Gal4>UAS-Sca3-Q78/CyO, and 4) shortGMR-Gal4>UASSca3-Q78/CyO.

\subsection{Survival Analysis}

For survival analysis, a genetic cross was set up to generate two types of progeny at equal frequency: one carrying a genotype of interest and the other, a sibling genotype, carrying a $\mathrm{CyO}$ balancer chromosome as an internal control group, similar to those described previously [22]. If the viability of a genotype is unaffected by either expressing a transgene, or by coexpressing two transgenes, the number of the progeny should be evenly divided by the two genotypes, which are recognized as carrying straight wings or carrying curly wings (determined by the dominant $C y$ mutation on the $C y O$ balancer). On the other hand, if the transgenic expression causes detrimental effects on the survival of a genotype, then straight-winged progeny would be significantly lower in number 
relative to the curly-winged ones. In the cases where synthetic lethality is induced, only curly-winged control progeny would be produced. Each of the crosses was repeated in vials of a triplet.

\subsection{Fluorescent Assay of a GFP Reporter Gene Activity}

Eye discs of the wandering third instar larvae were dissected out in a phosphate-buffer saline (PBS, $130 \mathrm{mM}$ $\mathrm{NaCl}, 7 \mathrm{mM} \mathrm{Na}_{2} \mathrm{HPO}_{4} \cdot 2 \mathrm{H}_{2} \mathrm{O}, 3 \mathrm{mM} \mathrm{NaH}_{2} \mathrm{PO}_{4} \cdot 2 \mathrm{H}_{2} \mathrm{O}$, pH 7.0) and the discs were examined immediately in PBS by using an Olympus microscope. Green fluorescent images were captured using a digital camera mounted on the microscope (Spot, Diagnostic Instrument, Inc.). Using the ImageJ software (the National Institutes of Health), each color image was split into three channels and the green channel was selected for quantitative analysis. The strongest fluorescent signals from the posterior edge of each disc (the first 3 - 5 rows of the developing ommatidia) were selected as Region-Of-Interest (ROI) and measured as Mean Grey Value in ImageJ.

\section{Results}

When induced by the GMR-Gal4/UAS system in the eyes, Dikar's expression caused compound external eye defects, including a rough surface and disrupted arrays of the bristles on the eye surface [22]. In addition, coexpressing Dikar and an expanded polyglutamine tract induced synthetic lethality at pupal stages. Because a conventional F1 genetic screen with a single-step approach could not be used to isolate synthetic lethal alleles, here we took a two-step approach. In the first step, UAS-alleles were randomly generated by $P$ element insertions and were selected for their capabilities of inducing eye defects when driven by a GMR-Gal4 driver. In the second step, the UAS-alleles were individually coexpressed with a protein containing an expanded polyglutamine tract to detect synthetic lethality.

\subsection{Isolation of Alleles That Disrupt Eye Development When Overexpressed}

To generate alleles that could be overexpressed by using GMR-Gal4/UAS, a genetically engineered $P$ element, $X P$, was used [22] [23]. Each end of the $X P$ element contains an UAS-expression construct that is orientated in the outward direction, so it is capable of overexpressing flanking endogenous genes. Using the $X P$ element to generate UAS-alleles that overexpress Drosophila genes is also extremely efficient, since the transposon preferentially inserts into the promoter regions of Drosophila genes [24]-[26].

We activated an XP element on Chromosome 3, d09108, by using a transposase source, Delta 2 - 3, to generate insertions randomly throughout the genome (Figure 1). A genetic screen was followed to identify UASalleles that disrupt eye development when overexpressed with a Gal4 driver, shortGMR-Gal4 [19] [27]. Briefly, in bottles, males carrying both $d 09108$ and Delta 2 - 3 were crossed to females carrying the shortGMR-Gal4 driver (Cross 1 in Figure 1). Single male progeny displaying eye defects, which were detected under a dissecting microscope, were selected from the mass mating. Each of these males was a candidate carrying a newly generated $X P$ element, which induces the eye defect by shortGMR-Gal4. Subsequently, each of the XP elements was mapped to the major chromosomes (Cross 2, Figure 1). Only the XP elements located on Chromosome 2 were retained for further investigation.

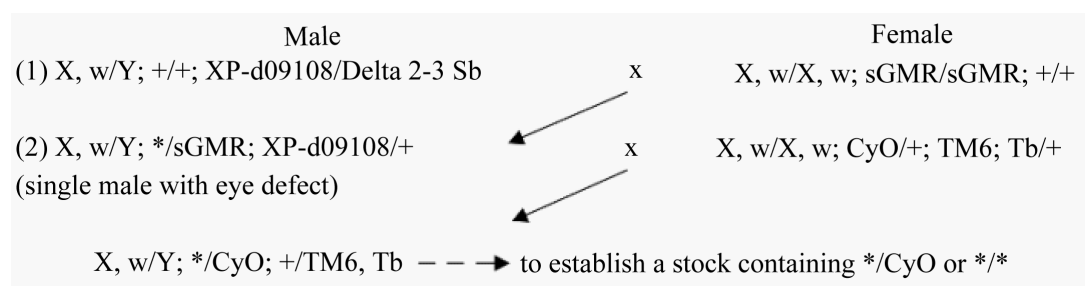

Figure 1. A scheme to isolate UAS-alleles that disrupt eye development when overexpressed. A UAS-carrying XP element, which is located on Chromosome 3, XP-d09108, was used as the starting element to transpose in the presence of a transposase source, Delta 2-3. After activating the $X P$ element, single male progeny displaying eye defect, which was induced by shortGMR-Gal4 (sGMR), was recovered from the first cross as a candidate of harboring a UAS-allele. Insertions on Chromosome 2 were of interest. Such a newly inserted XP on Chromosome 2, indicated by an asterisk, was identified when no progeny with defective eyes were produced from the second cross. Subsequently, individual stocks were established to carry an XP insertion on Chromosome 2. 


\subsection{Eye Defects Associated with the Isolated UAS-Alleles}

From the genetic screen in Figure 1, five strains carrying XP insertions on Chromosome 2 were obtained. The eye defects, which were induced by overexpressing the UAS-alleles using shortGMR-Gal4, could be roughly divided into several types, including uneven surface, oily-looking surface, eye color change, irregular distribution of the eye colors, and loss of the external bristles. The defects were often superimposed on each other, resulting in compound eye phenotypes as shown in Figure 2(a).

In addition to shortGMR-Gal4, we examined the effect of another related Gal4 driver, longGMR-Gal4. Both of the GMR drivers contain pentamerized glass-binding sites, enabling them to express the Gal4 protein in the eyes [19] [27]. While shortGMR-Gal4 carries slightly shorter glass-binding core sites of $28 \mathrm{bp}$, longGMR-Gal4 carries 39bp glass-binding sites. shortGMR-Gal4 induced more severe eye phenotypes than longGMR-Gal4 when used in experiments to drive gene expression that caused eye defects. Using a GFP reporter gene, UAS$2 x E G F P$ [20], we examined the relative expression levels of two GMR-Gal4 drivers in the larval eye imaginal discs. We constructed a pair of genotypes expressing GFP, shortGMR-Gal4>UAS-2xEGFP and longGMR-Gal4 > UAS-2xEGFP. The results showed that the mean GFP signal level of the shortGMR-Gal4>UAS-2xEGFP genotype was approximately 8.3 times higher than that of the longGMR-Gal4>UAS-2xEGFP genotype (59.7 \pm 14.9 vs. $7.2 \pm 2.5, \mathrm{p}<0.001$, Figure 3 ).

We then examined the eye defects induced by overexpressing the five UAS-strains with either shortGMRGal4 or longGMR-Gal4. The results showed that the degree to which eye defects could be induced by the two drivers is correlated with the level of the drivers' strength. Overall, using the stronger shortGMR-Gal4 driver induced more severe eye defects than the longGMR-Gal4 driver (Figure 2(a) and Figure 2(b)). For example, the eyes of a genotype carrying UAS-8 showed rougher, or bumpier, surfaces when driven by shortGMR-Gal4 than longGMR-Gal4. The eye surface of the genotype expressing UAS-22 displayed a severer loss of the bristles when driven by shortGMR-Gal4 than longGMR-Gal4. In fact, even in a wild type background, the shortGMR-Gal4 driver by itself induced a defect on the eye surface seen as unevenly distributed bristles under a higher magnification, while the eye of the longGMR-Gal4 appeared mostly normal (WT, Figure 2(a) and Figure 2(b)).

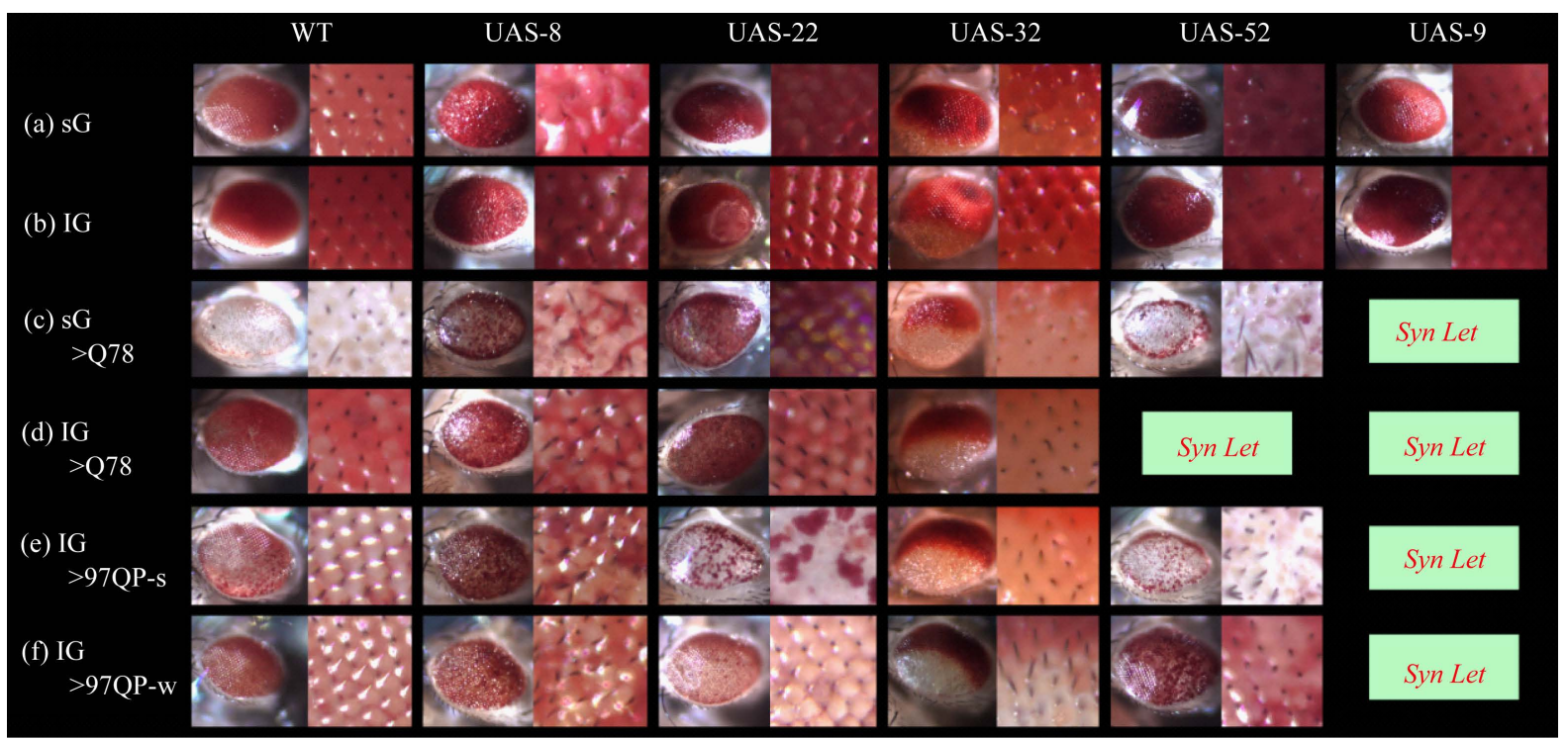

Figure 2. Eye defects associated with five UAS-alleles. (a) Expression of UAS-alleles with shortGMR-Gal4 (sG). (b) Expression of UAS-alleles with longGMR-Gal4 (lG). (c) Coexpression of each UAS-allele and UAS-Sca3-Q78 with shortGMR-Gal4. sG>Q78, shortGMR-Gal4 drives UAS-Sca3-Q78. (d) Coexpression of each UAS-allele and UAS-Sca3-Q78 with longGMR-Gal4. IG>Q78 indicates that longGMR-Gal4 drives UAS-Sca3-Q78. (e) Coexpression of each UAS-allele and UAS-httex1p-97QP-s with longGMR-Gal4. IG>97QP-s indicates that longGMR-Gal4 drives UAS-httex1p-97QP-s. (f) Coexpression of each UAS-allele and UAS-httex1p-97QP-w with longGMR-Gal4. IG>97QP-w indicates that longGMR-Gal4 drives UAS-httex1p-97QP-w. UAS-httex1p-97QP-s is a stronger allele than that of UAS-httex1p-97QP-w [22]. Syn Let indicates a synthetic lethal genotype. 


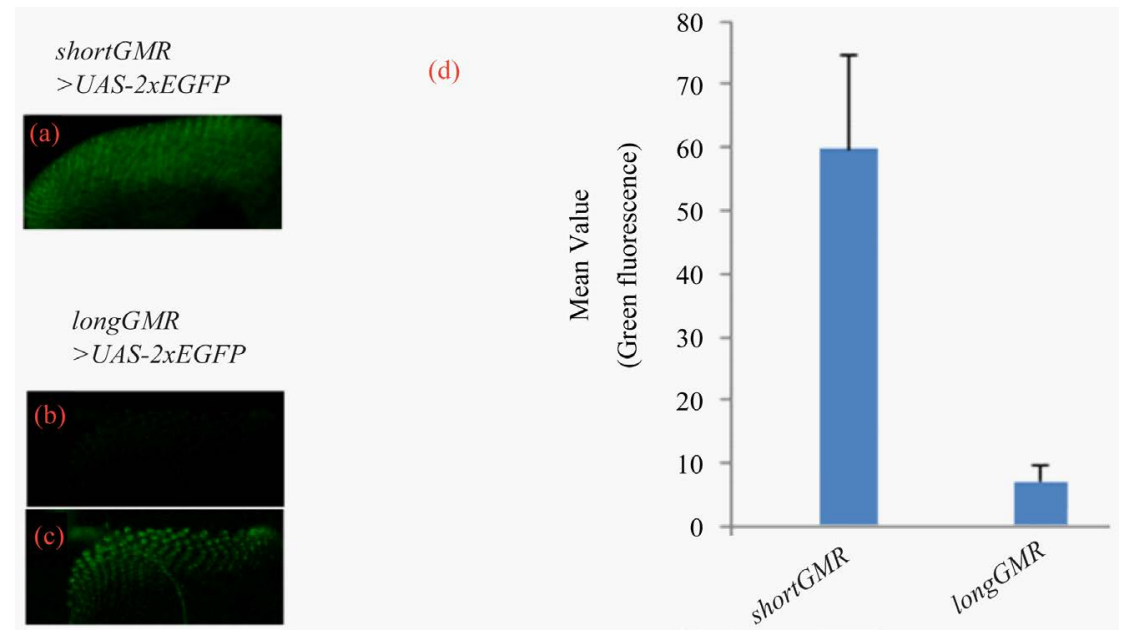

Figure 3. Activity of the shortGMR-Gal4 and longGMR-Gal4 drivers in eye imaginal discs. (a) (b) A GFP reporter gene, UAS-2xEGFP, was used to examine activities of two GMR-Gal4 drivers. Representative images were taken from late third instar larval imaginal eye discs of two genotypes, shortGMR-Gal4>UAS-2xEGFP and longGMR-Gal4> $U A S-2 x E G F P$. The signals from expressing UAS-2xEGFP were captured under identical exposure conditions (Panel (a) and (b)) to allow a direct comparison of the driving strengths between shortGMR-Gal4 and longGMR-Gal4. (c) The image was identical to that in Panel B, except that it was digitally enhanced by increasing the brightness level. (d) A quantitative analysis of the EGFP reporter signals. The green fluorescent intensity (Mean value) from the shortGMR-Gal4>UAS-2xEGFP genotype (20 discs) was significantly stronger than that of longGMR-Gal4>UAS-2xEGFP (22 discs) ( $\mathrm{p}<0.001$, twotailed Student's $t$-test). The Mean Grey Value of the fluorescent strength for each disc, which was measured using Image (see Methods), was used to calculate the mean value for each of the genotypes.

\subsection{Expressing the UAS-Alleles by Two GMR-Gal4 Drivers Showed Very Little Negative Effects on Survival}

To ask if expression of the UAS-alleles affects viability in Drosophila, we expressed the individual UAS-alleles with shortGMR-Gal4 and assayed for their survival. To determine the survival rate, a genetic cross was used to produce a pair of the sibling genotypes. One genotype expressed the UAS-allele, while the other was a wild type control containing a balancer chromosome, $\mathrm{CyO}$. If a UAS-allele expression induced no detrimental effect on viability, the two sibling genotypes would be produced at the same frequency. On the other hand, if the expression caused a viability problem, the control genotype would be produced at a higher frequency than the sibling genotype expressing the UAS-allele. For all of the UAS-alleles, the results show that progeny of the genotype expressing an individual UAS-allele were produced either at a frequency comparable to the internal wild type control (approximately 50\%), or at a high frequency than the control sibling genotype ( $p>0.05$, Figure 4(a)). Similar results were obtained when the longGMR-Gal4 driver was used to express the individual UAS-alleles ( $\mathrm{p}>$ 0.05, Figure 4(b)). Thus, none of these alleles, when driven by either of the GMR-Gal4 drivers, were detrimental to viability.

\subsection{Coexpressing the UAS-Alleles with an Expanded Polyglutamine Tract Induced Survival Problems including Synthetic Lethality}

The UAS-alleles were individually coexpressed with a transgene encoding a polyglutamine tract consisting of 97 glutamine residues, UAS-httex1p-97QP-s [22]. These tests revealed that some of the transgenes when coexpressed were detrimental to the survival, giving rise to reduced viability and even synthetic lethality. Shown in Figure 5(a), coexpressingUAS-22 and UAS-httex1p-97QP-s with longGMR-Gal4 induced a decreased survival rate, from $52.7 \%$ of the control to $44.2 \%(\mathrm{p}=0.024)$, while coexpressingUAS-52 and UAS-httex $1 p-97 Q P-s$ caused a sharp decline on the survival rate to only $16.6 \%(\mathrm{p}=0.003)$. The strongest effect from the coexpressing tests was a synthetic lethality phenotype that was induced by coexpressingUAS-9 and UAS-httex1p-97QP-s with 


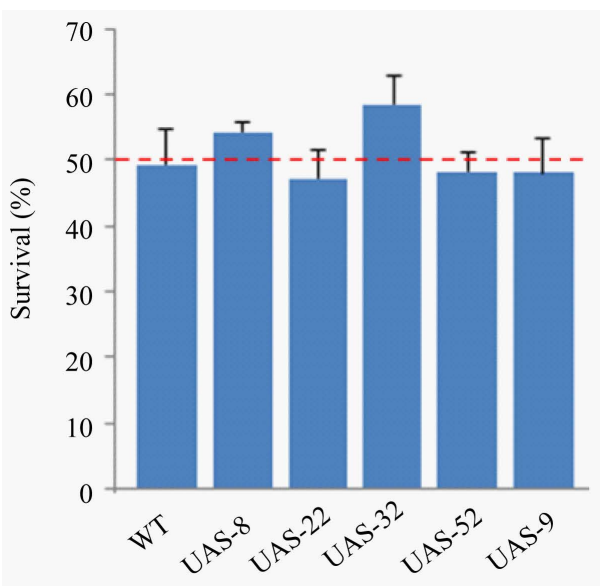

(a) longGMR-Gal4

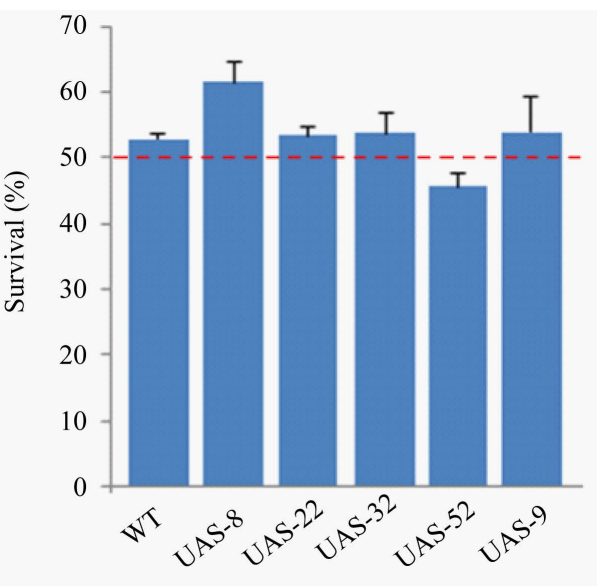

(b) shortGMR-Gal4

Figure 4. Survival rates of five genotypes expressing UAS-alleles with either of the GMR-Gal4 drivers. (a) Expression driven by shortGMR-Gal4. (b) Expression driven by longGMR-Gal4. Each genotype in the figure was generated from a cross, along with another sibling genotype containing a $\mathrm{CyO}$ balancer. Thus, from each cross, the genotype that expressed a transgene accounted for approximately $50 \%$ of the total progeny (red dashed line) when the overexpression did not weaken the survival. However, when a detrimental effect was induced by expressing the UAS-gene, the $C y^{+}$progeny should account for significantly less than $50 \%$ of the total progeny from the cross. WT, a wild type control.

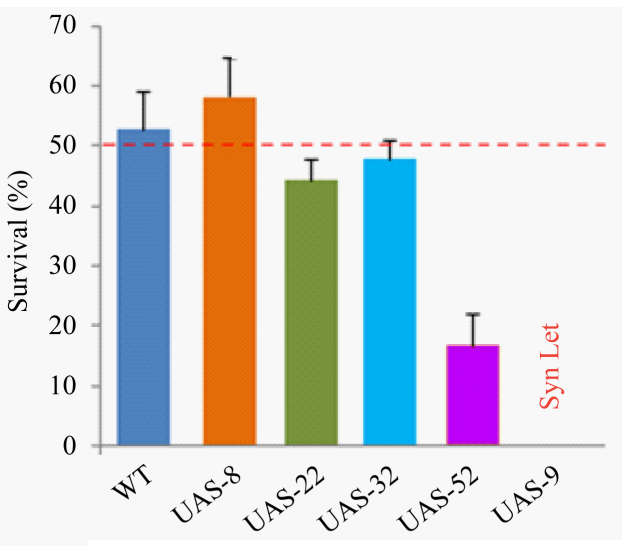

(a) longGMR-Gal4

$>$ UAS-httex1p-97QP-s

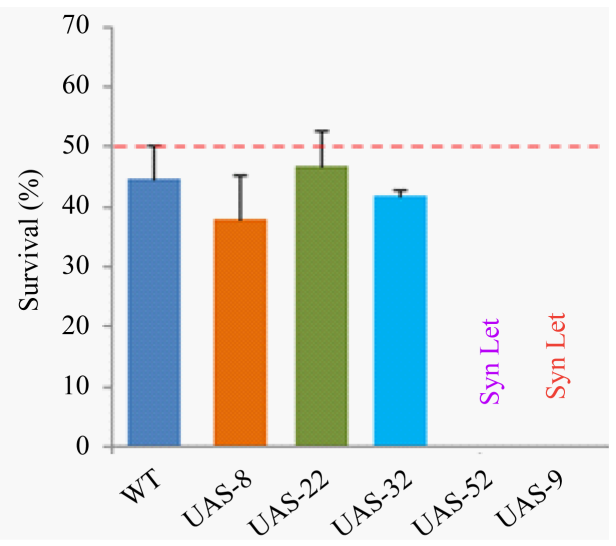

(b) shortGMR-Gal4

$>$ UAS-Sca3-Q78

Figure 5. Survival rates of genotypes coexpressing the UAS-alleles and two expanded polyglutamine proteins with the longGMR-Gal4 driver. (a) Coexpression of each UAS-allele and UAS-httex1p-97QP-s with longGMR-Gal4; (b) Coexpression of each UAS-allele and UAS-Sca3-Q78 with longGMR-Gal4. WT, a wild type control. Syn Let, a synthetic lethal phenotype.

longGMR-Gal4. We also coexpressed the UAS-alleles with another transgene, UAS-Sca3-Q78, which encodes a polyglutamine tract consisting of 78 glutamine residues [18]. Data from this later coexpression series showed that UAS-9, or UAS-52, when coexpressed with UAS-Sca3-Q78, each generated a synthetic lethality (Figure 5(b)).

The UAS-httex1p-97QP-s and UAS-Sca3-Q78 transgenes do not share any homologous sequences, despite of each encoding an expanded polyglutamine tract. This is because the expanded polyglutamine tracts are encoded in two different ways. While alternate CAA and CAG codons were incorporated in UAS-httex $1 p$-97QP, only the CAG repeats are present in UAS-Sca3-Q78 [18] [22] [28]. In addition to the differential codon usages, the encoding protein domains flanking the tracts are also unrelated between httex1p-97QP and Sca3-Q78. Since the only obvious similarity between the transgenes is that each of them encodes a toxic polyglutamine tract, the 
synthetic lethality from both of the coexpression tests was most likely mediated through the expanded polyglutamine tracts. To test this possibility, an additional coexpression experiment was carried out by using a third transgene encoding only an expanded polyglutamine tract without flanking domains, UAS-41Q [29]. CoexpressingUAS-9 and UAS-41Q with longGMR-Gal4 also induced synthetic lethality, providing further supporting evidence that the observed synthetic lethality is mediated through the neurotoxic polyglutamine tract.

As a control, we also examined UAS-Sca3-Q27, which encodes a non-toxic tract of 27 glutamine residues. It did not cause viability issues when coexpressed with UAS-9 by longGMR-Gal4. Taken together, these results indicated that the synthetic lethality of UAS-9 was mediated through a toxic polyglutamine tract.

\subsection{Survival Rate and the GMR-GAL4/UAS Activity}

The UAS-alleles were isolated for their capabilities of inducing eye defects when overexpressed. Overall, the severity of these eye defects was correlated with the strength of the GMR-Gal4 drivers (Figure 2(a) and Figure 2(b)). To investigate if the strength of the Gal4 drivers in the eyes was also correlated with the survival rate of a genotype that coexpresses an UAS-allele and an expanded polyglutamine tract, we used the pair of GMR-Gal4 drivers, longGMR-Gal4 and shortGMR-Gal4. The results in Figure 6 show the survival rates of five genotypes coexpressing the UAS-alleles individually with UAS-Sca3-Q78 by using either longGMR-Gal4, or shortGMRGal4. Expressing UAS-Sca3-Q78 in a wild type background by the stronger shortGMR-Gal4 driver appeared to induce a slightly lower survival rate than that of longGMR-Gal4 (0.449 \pm 0.047 vs. $0.366 \pm 0.031, \mathrm{p}=0.05)$. CoexpressingUAS-Sca3-Q78 and UAS-8 by shortGMR-Gal4 may also slightly reduce the survival rate (0.391 \pm 0.062 vs. $0.312 \pm 0.018, \mathrm{p}=0.08$ ). However, coexpressingUAS-22 and UAS-Sca3-Q78 by shortGMR-Gal4 induced a significantly lower survival rate than that of longGMR-Gal4 (0.466 \pm 0.050 vs. $0.213 \pm 0.016, p=0.01)$. Thus, the results from coexpressing each of these UAS-alleles and an expanded polyglutamine protein by shortGMR-Gal4 showed a tendency of lowering the survival rates by a stronger Gal4 driver in the eyes than a weaker one.

However, coexpressing other UAS-alleles with UAS-Sca3-Q78 by shortGMR-Gal4 resulted in increased survival rates than that of longGMR-Gal4 (Figure 6). When UAS-32 and UAS-Sca3-Q78 were coexpressed by shortGMR-Gal4, the survival rate was significantly higher than that of the longGMR-Gal4 (0.424 \pm 0.009 vs. $0.528 \pm 0.034, \mathrm{p}=0.027$ ). Furthermore, when UAS-52 was coexpressed with UAS-Sca3-Q78 by shortGMRGal4, a significant fraction of the genotype survived to adulthood (21.2\%), while the coexpression by longGMRGal4 induced a synthetic lethality ( $\mathrm{p}=0.002$, Figure 6 ). Synthetic lethality was seen when UAS-9 was coexpressed with UAS-Sca3-Q78 by using either longGMR-Gal4 or shortGMR-Gal4.

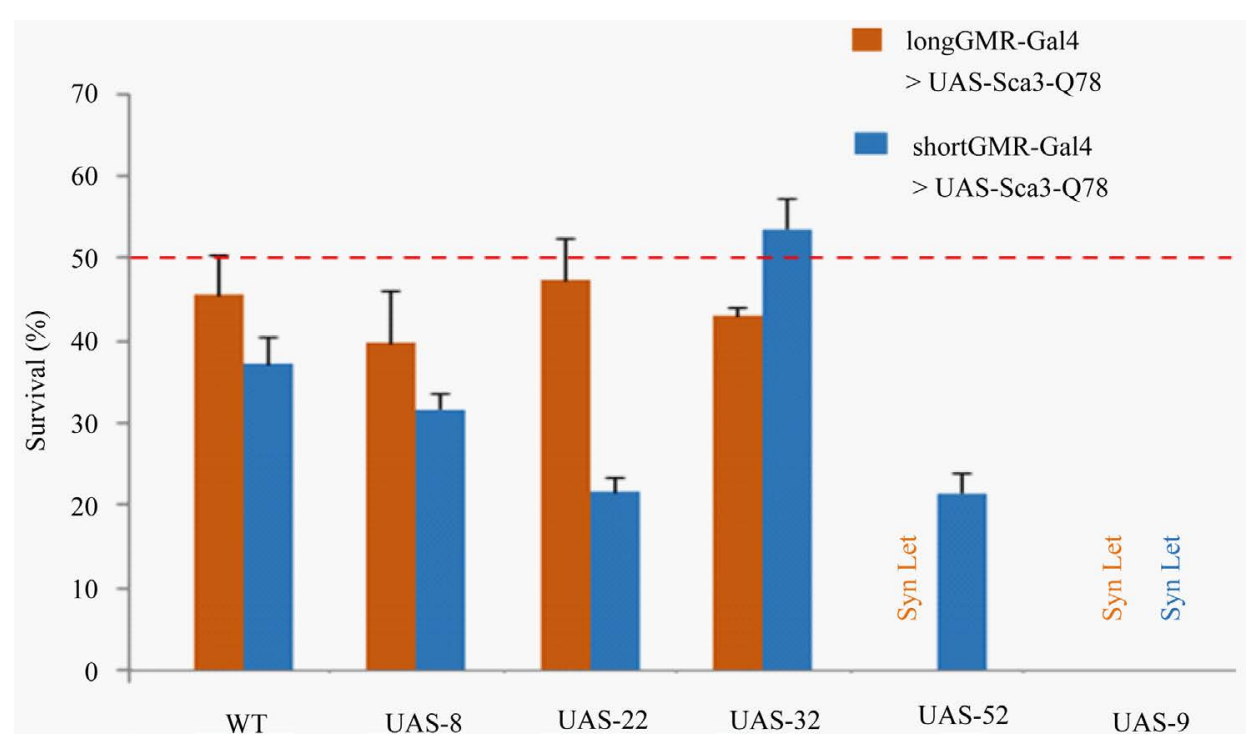

Figure 6. Survival rates of genotypes coexpressing the UAS alleles and UAS-Sca-Q78 with either the longGMR-Gal4 or shortGMR-Gal4 driver. WT, a wild type control; Syn Let, a synthetic lethal phenotype. 


\subsection{Survival Rates and the Strength of Alleles Encoding an Expanded Polyglutamine Protein}

In addition to examining the effects on survival rate from drivers, we also studied the effects from two alleles that express an extended polyglutamine tract, UAS-httex1p-97QP-w (an eye weak allele) and UAS-httex1p97QP-s (an eye strong allele) [22]. When driven by longGMR-Gal4, UAS-httex1p-97QP-w caused slightly less color loss than that of UAS-httex1p-97QP-s (Figure 2). As shown in Figure 7, coexpressing all five UAS-alleles individually with $U A S-h t t e x 1 p-97 Q P-w$ had nearly the same effects on the survival rates as those of UAShttex1p-97QP-s (p > 0.05). An exception was UAS-Dikar that was isolated in our previous experiments [22]. While coexpressing UAS-Dikar and UAS-httex1p-97QP-s induced a synthetic lethal phenotype, the vast majority of the genotype coexpressing UAS-Dikar and UAS-httex1p-97QP-w survived to adulthood (Figure 7, p < 0.001). Thus, UAS-Dikar was the only allele that displayed a correlation of its responses to UAS-polyQ alleles' strength in the eyes.

\subsection{Survival Rates and Temperature}

Temperature is known to affect the activity of the Gal4/UAS system in Drosophila, with a range from $29^{\circ} \mathrm{C}$ (strong expression output) to $18^{\circ} \mathrm{C}$ (weak expression output) [30]. Using a GFP reporter gene, we examined the expression levels of the longGMR-Gal4>UAS-2xEGFP genotype grown at $23^{\circ} \mathrm{C}$ and $25^{\circ} \mathrm{C}$. The results showed that the relative GFP signal intensity of the larval eye discs grown at $25^{\circ} \mathrm{C}$ is approximately $84 \%$ higher than that at $23^{\circ} \mathrm{C}(24.5 \pm 7.9$ vs. $13.3 \pm 4.3, \mathrm{p}=0.002)$.

We then asked if the survival rates of the genotypes coexpressing the UAS-strains and an expanded polyglutamine protein also show a temperature dependency. As shown in Figure 8(a), at $25^{\circ} \mathrm{C}$, UAS-52 caused severe viability problems when it was coexpressed with the UAS-httex $1 p-97 Q P-w$ allele with longGMR-Gal4. At $23^{\circ} \mathrm{C}$, a significantly less severe effect on the survival rate was observed $\left(0.461 \pm 0.046\right.$ at $25^{\circ} \mathrm{C}$ vs. $0.166 \pm 0.032$ at $\left.23^{\circ} \mathrm{C}, \mathrm{p}<0.001\right)$. A more striking effect of the temperature shift was seen for UAS-9. While its coexpression at $25^{\circ} \mathrm{C}$ with UAS-httex $1 p-97 Q P-w$ induced synthetic lethality, more than $50 \%$ of the coexpressing genotype survived to adulthood at $23^{\circ} \mathrm{C}(0.274 / 0.5$ vs. $0, \mathrm{p}<0.001$, Figure $8(\mathrm{a}))$.

It is difficult to assess if there is a correlation between the survival rates and the compound eye defects from the coexpression experiments, since expressing each of these UAS-alleles alone in the eyes causes various eye defects (Figure 2). Moreover, at $25^{\circ} \mathrm{C}$, some of the coexpressing genotypes were synthetic lethal and thus their eye phenotypes remain unknown. UAS-9 is an extreme case, because, at $25^{\circ} \mathrm{C}$, coexpressingUAS-9 resulted in

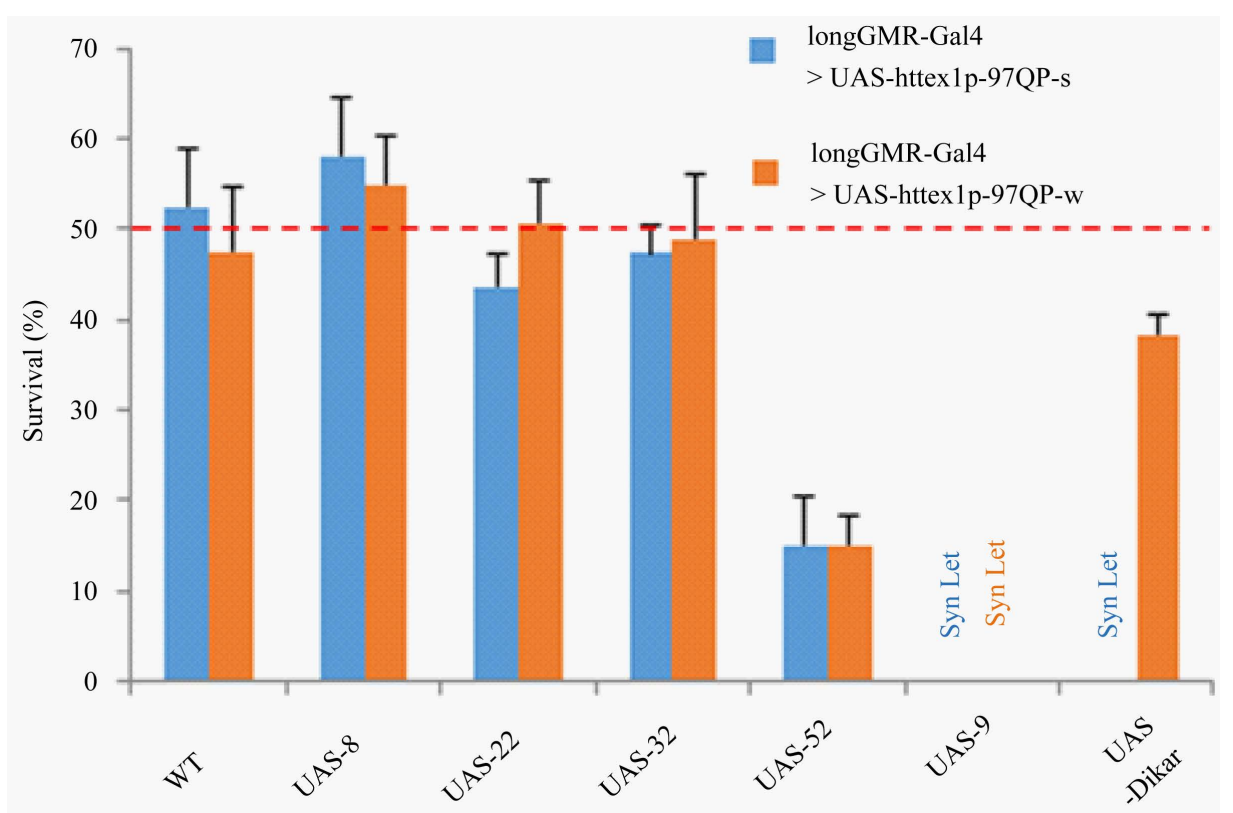

Figure 7. Survival rates of genotypes coexpressing a UAS-allele and either UAS-httex1p-97QP-s, or UAS-httex1p-97QP-s, with longGMR-Gal4. WT, a wild type control; Syn Let, a synthetic lethal phenotype. 


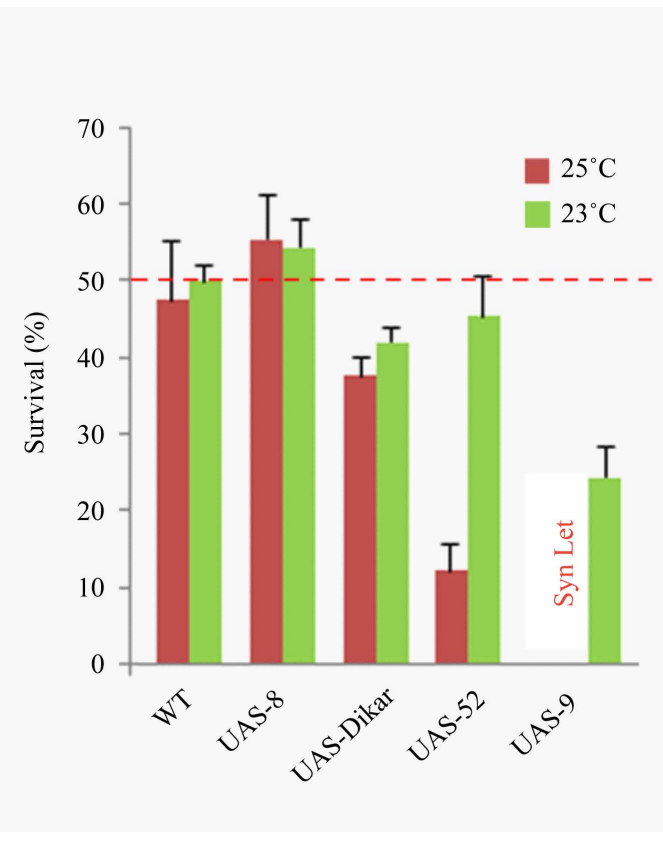

(a)

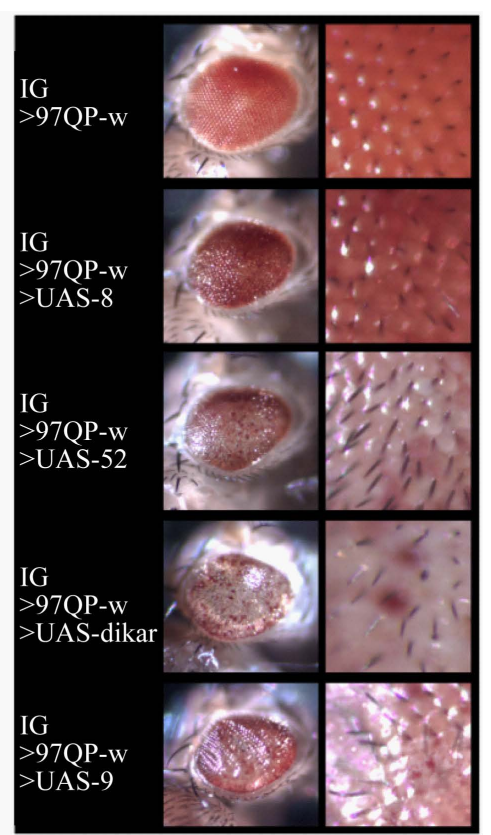

(b)

Figure 8. Survival and eye defects of genotypes coexpressing the UAS-alleles and UAS-httex $1 p-97 Q P-w$ with longGMR-Gal4 at $25^{\circ} \mathrm{C}$ and $23^{\circ} \mathrm{C}$. (a) Survival of genotypes coexpressing the UAS-alleles and UAShttex1p-97QP-w with longGMR-Gal4; (b) Adult eye defects resulting from coexpressing a UAS-allele and UAS-httex1p-97QP-w with longGMR-Gal4 at $23^{\circ} \mathrm{C}$. WT, a wild type control; Syn Let, a synthetic lethal phenotype; IG, longGMR-Gal4; UAS-97QP-w, UAS-httex1p-97QP-w.

synthetic lethality in all of the tests (Figure 2). At $23^{\circ} \mathrm{C}$, the surviving adults of coexpressingUAS-9 and UAS$h$ ttex1p-97QP-w with longGMR-Gal4 provided an opportunity to reveal the coexpressing eye defects. As shown in Figure 8(b), the adult eyes of this genotype displayed loss of eye color, missing bristles, and an uneven eye surface. In addition, the eye surface was often collapsed, indicating structural damages underneath. Thus, it appears that the UAS-9 allele, which induced synthetic lethality under all of the tested coexpression conditionsat $25^{\circ} \mathrm{C}$, caused the most severe eye damage when coexpressed with UAS-httex $1 p-97 Q P$ - $w$ by long-GMR-Gal4 at $23^{\circ} \mathrm{C}$.

\section{Discussion}

The Drosophila eye consists of approximately 750 reiterated units, referred to as the ommatidia. Some mutations cause minor perturbation to the eye formation, which could be seen as slightly disordered arrays of the ommatidia, or a rough eye, directly under a dissecting microscope. Thus, the Drosophila eye is highly sensitive to mutations and the corresponding phenotypes are readily visible. The sensitivity and efficiency of using the eye as a tool to monitor genetic modifications have made the eye a favorite model in investigations of genetic interactions. Furthermore, since adult flies with severe eye defects, which were induced by mutations such as eya (eyes absent) were viable [31], the eye model is largely regarded as unbiased in genetic screens, allowing the isolation of both weak and strong mutations that influence eye formation.

To investigate gene activity and genetic pathways, expressing genes in the developing Drosophila eyes by using GMR-Gal4/UAS has been employed more frequently than any other expression systems [30] [32]. This system has facilitated the identification and characterization of large collections of genes and genetic pathways that interact with the expanded polyglutamine proteins [29] [33]-[36]. However, despite the fact that GMR-Gal4/UAS operates predominantly in the eyes, the system triggers "leaky" expression in tissue types other than in the eyes. For example, the transcription factor that regulates GMR-Gal4, the glass protein, has been found in a number of non-eye tissue types [37]-[39]. Moreover, the glass gene is essential for the larval development, because loss-offunction glass mutants cause developmental failure at larval stages [38] [40]-[42]. In addition, the isolation of the Drosophila synthetic lethal Dikar gene [22] and additional synthetic lethal alleles described in this report 
have raised a possibility that GMR-Gal4/UAS is biased against the isolation of strongest interacting modifiers, i.e., the synthetic lethal alleles.

Due to its innate toxic nature, an expanded polyglutamine tract may generate a lethal-prone condition, frequently giving rise to synthetic lethality when the genetic background is modified. It is possible that the GMRGal4 drivers operate in a non-eye cell type that is extremely sensitive to the neural toxin. Thus, the seemingly trivial yet toxic expression could lead to a near-catastrophic condition to the cells that are essential for viability, though the leaky non-eye expression is insignificant when compared to that of the primary activity in the eyes. Consistent with this hypothesis, our results indicate that the synthetic lethality phenomenon is unrelated to the primary GMR-Gal4's activity in the eyes. First, Expressing UAS-9 in the eyes caused eye defects that are compatible to those of several other UAS-strains, such as UAS-8. UAS-9 induced synthetic lethality in all of the coexpression assays, while UAS-8 had very little, if any, effects in the assays. Second, though the shortGMR-Gal4 activity is more than 8 times higher than that of longGMR-Gal4 in the developing eyes, its effects on survival was weaker than longGMR-Gal4 in a set of the coexpressing assays involving UAS-52 and UAS-Sca3-Q78 (21.2\% survival rate vs. a synthetic lethality) (Figure 6).

This survey showed that GMR-Gal4 would allow the strongest modifiers, i.e., the synthetic-lethal alleles, to escape detections in the conventional genetic screens. For example, UAS-52 would likely have escaped the detections, since the assays mostly generated coexpression genotypes that were either synthetic lethal or low in viability. Another example is UAS-Dikar, which could have gone unnoticed during detection as, coexpressingUAS-Dikar and an expanded polyglutamine tract nearly always resulted in synthetic lethality [22], it would have had almost certainly escaped the detections. UAS-9 is an extreme example, which would certainly be excluded from the conventional screens. The genotypes coexpressingUAS-9 and any one of the four UAS-polyglutamine transgenes (UAS-httex1p-97QP-w, UAS-httex1p-97QP-s, UAS-Sca3-Q78, or UAS-41Q) with either of the two GMR-Gal4 drivers (longGMR-Gal4 or shortGMR-Gal4) were synthetic lethal. Surviving progeny of a coexpression genotype, longGMR-Gal4>UAS-9 + UAS-httex1p-97QP-w, were seen only after the rearing temperature was lowered to $23^{\circ} \mathrm{C}$ from $25^{\circ} \mathrm{C}$, a condition that reduced the Gal4/UAS activity by roughly $50 \%$ and presumably lowered the activity of the cell death pathways, too.

In spite of having established many models to develop therapeutic interventions for the expanded polyglutamine diseases, the critical targets of the treatments including the major cellular components, or physiological pathways, remain elusive [3] [6] [7] [16] [43] [44]. Synthetic lethality reveals an essential genetic interaction that provides a buffering mechanism [45]. The synthetic-lethal alleles likely belong to interacting genes that are not only stronger than the known modifiers, but also act in the same biochemical pathway in which the expanded polyglutamine tract induces cellular toxicity, or in a parallel buffering pathway that operates in the same physiological process [46]-[48]. It is also possible that these alleles interact with an expanded polyglutamine tract at early stages of the model disease development, which could disrupt multiple physiological processes and amplify the toxicity. Since results from studying stronger modifiers, essential interactions, and earlier players in the disease development may be used to better target the diseases, isolation and characterization of the synthetic-lethal alleles could play pivotal roles in developing therapeutic strategies. In addition to the synthetic lethality associated with expressing a toxic polyglutamine tract, similar synthetic lethality may also be commonly associated with expressing other acute toxic disease proteins in model organisms, since it is still difficult for the current heterologous gene expression technology to precisely target a specific cell type without the leaky side effects. To identify the synthetic-lethal alleles, however, exploring alternative approaches to the conventional F1 genetic screens, such as the two-step design used in this study, would be necessary.

\section{Conclusion}

Our results suggest that the toxic polyglutamine tract induces an acute condition that could easily leads to cell death when strong interacting factors are present. The synthetic lethal alleles may be more common that thought, and might have escaped the conventional genetic detection methods, due the synthetic lethality. Future studies into the mechanistic aspects of the synthetic lethal alleles will provide insight into the critical physiological pathways where the synthetic lethal alleles interact with the expanded polyglutamine proteins to cause neurodegenerative cell death.

\section{Fund}

This study was supported by grants from University of Connecticut. 


\section{References}

[1] Orr, H.T. and Zoghbi, H.Y. (2007) Trinucleotide Repeat Disorders. Annual Review of Neuroscience, 30, 575-621. http://dx.doi.org/10.1146/annurev.neuro.29.051605.113042

[2] Yu, Z. and Bonini, N.M. (2011) Modeling Human Trinucleotide Repeat Diseases in Drosophila. International Review of Neurobiology, 99, 191-212. http://dx.doi.org/10.1016/b978-0-12-387003-2.00008-2

[3] Figiel, M., Szlachcic, W.J., Switonski, P.M., Gabka, A. and Krzyzosiak, W.J. (2012) Mouse Models of Polyglutamine Diseases: Review and Data Table. Part I. Molecular Neurobiology, 46, 393-429. http://dx.doi.org/10.1007/s12035-012-8315-4

[4] Blum, E.S., Schwendeman, A.R. and Shaham, S. (2012) PolyQ Disease: Misfiring of a Developmental Cell Death Program? Trends in Cell Biology, 23, 168-174.

[5] Costa Mdo, C. and Paulson, H.L. (2012) Toward Understanding Machado-Joseph Disease. Progress in Neurobiology, 97, 239-257. http://dx.doi.org/10.1016/j.pneurobio.2011.11.006

[6] Knott, A.B., Perkins, G., Schwarzenbacher, R. and Bossy-Wetzel, E. (2008) Mitochondrial Fragmentation in Neurodegeneration. Nature Reviews Neuroscience, 9, 505-518. http://dx.doi.org/10.1038/nrn2417

[7] Sugars, K.L. and Rubinsztein, D.C. (2003) Transcriptional Abnormalities in Huntington Disease. Trends in Genetics, 19, 233-238. http://dx.doi.org/10.1016/S0168-9525(03)00074-X

[8] Mangiarini, L., Sathasivam, K., Seller, M., Cozens, B., Harper, A., Hetherington, C., Lawton, M., Trottier, Y., Lehrach, H., Davies, S.W., et al. (1996) Exon 1 of the HD Gene with an Expanded CAG Repeat Is Sufficient to Cause a Progressive Neurological Phenotype in Transgenic Mice. Cell, 87, 493-506. http://dx.doi.org/10.1016/S0092-8674(00)81369-0

[9] Marsh, J.L., Walker, H., Theisen, H., Zhu, Y.Z., Fielder, T., Purcell, J. and Thompson, L.M. (2000) Expanded Polyglutamine Peptides Alone Are Intrinsically Cytotoxic and Cause Neurodegeneration in Drosophila. Human Molecular Genetics, 9, 13-25. http://dx.doi.org/10.1093/hmg/9.1.13

[10] Brignull, H.R., Moore, F.E., Tang, S.J. and Morimoto, R.I. (2006) Polyglutamine Proteins at the Pathogenic Threshold Display Neuron-Specific Aggregation in a Pan-Neuronal Caenorhabditiselegans Model. Journal of Neuroscience, 26, 7597-7606. http://dx.doi.org/10.1523/JNEUROSCI.0990-06.2006

[11] Ellerby, L.M., Andrusiak, R.L., Wellington, C.L., Hackam, A.S., Propp, S.S., Wood, J.D., Sharp, A.H., Margolis, R.L., Ross, C.A., Salvesen, G.S., et al. (1999) Cleavage of Atrophin-1 at Caspase Site Aspartic Acid 109 Modulates Cytotoxicity. Journal of Biological Chemistry, 274, 8730-8736. http://dx.doi.org/10.1074/jbc.274.13.8730

[12] Goti, D., Katzen, S.M., Mez, J., Kurtis, N., Kiluk, J., Ben-Haiem, L., Jenkins, N.A., Copeland, N.G., Kakizuka, A., Sharp, A.H., et al. (2004) A Mutant Ataxin-3 Putative-Cleavage Fragment in Brains of Machado-Joseph Disease Patients and Transgenic Mice Is Cytotoxic above a Critical Concentration. Journal of Neuroscience, 24, 10266-10279. http://dx.doi.org/10.1523/JNEUROSCI.2734-04.2004

[13] Jung, J., Xu, K.Q., Lessing, D. and Bonini, N.M. (2009) Preventing Ataxin-3 Protein Cleavage Mitigates Degeneration in a Drosophila Model of SCA3. Human Molecular Genetics, 18, 4843-4852. http://dx.doi.org/10.1093/hmg/ddp456

[14] van Ham, T.J., Breitling, R., Swertz, M.A. and Nollen, E.A. (2009) Neurodegenerative Diseases: Lessons from Genome-Wide Screens in Small Model Organisms. EMBO Molecular Medicine, 1, 360-370. http://dx.doi.org/10.1002/emmm.200900051

[15] Blum, E.S., Schwendeman, A.R. and Shaham, S. (2013) PolyQ Disease: Misfiring of a Developmental Cell Death Program? Trends in Cell Biology, 23, 168-174. http://dx.doi.org/10.1016/j.tcb.2012.11.003

[16] Switonski, P.M., Szlachcic, W.J., Gabka, A., Krzyzosiak, W.J. and Figiel, M. (2012) Mouse Models of Polyglutamine Diseases in Therapeutic Approaches: Review and Data Table. Part II. Molecular Neurobiology, 46, 430-466. http://dx.doi.org/10.1007/s12035-012-8316-3

[17] Jackson, G.R. (2008) Guide to Understanding Drosophila Models of Neurodegenerative Diseases. PLoS Biology, 6, e53.

[18] Warrick, J.M., Paulson, H.L., Gray-Board, G.L., Bui, Q.T., Fischbeck, K.H., Pittman, R.N. and Bonini, N.M. (1998) Expanded Polyglutamine Protein Forms Nuclear Inclusions and Causes Neural Degeneration in Drosophila. Cell, 93, 939-949. http://dx.doi.org/10.1016/S0092-8674(00)81200-3

[19] Wernet, M.F., Labhart, T., Baumann, F., Mazzoni, E.O., Pichaud, F. and Desplan, C. (2003) Homothorax Switches Function of Drosophila Photoreceptors from Color to Polarized Light Sensors. Cell, 115, 267-279. http://dx.doi.org/10.1016/S0092-8674(03)00848-1

[20] Halfon, M.S., Gisselbrecht, S., Lu, J., Estrada, B., Keshishian, H. and Michelson, A.M. (2002) New Fluorescent Protein Reporters for Use with the Drosophila Gal4 Expression System and for Vital Detection of Balancer Chromosomes. Genesis, 34, 135-138. http://dx.doi.org/10.1002/gene.10136 
[21] Li, L.B. and Bonini, N.M. (2010) Roles of Trinucleotide-Repeat RNA in Neurological Disease and Degeneration. Trends in Neurosciences, 33, 292-298. http://dx.doi.org/10.1016/j.tins.2010.03.004

[22] Zhang, P., Wang, Q.M., Hughes, H. and Intrieri, G. (2014) Synthetic Lethality Induced by a Strong Drosophila Enhancer of Expanded Polyglutamine Tract. Open Journal of Genetics, 4, 300-315. http://dx.doi.org/10.4236/ojgen.2014.44028

[23] Thibault, S.T., Singer, M.A., Miyazaki, W.Y., Milash, B., Dompe, N.A., Singh, C.M., Buchholz, R., Demsky, M., Fawcett, R., Francis-Lang, H.L., et al. (2004) A Complementary Transposon Tool Kit for Drosophila melanogaster using P and Piggy Bac. Nature Genetics, 36, 283-287. http://dx.doi.org/10.1038/ng1314

[24] Spradling, A.C., Stern, D.M., Kiss, I., Roote, J., Laverty, T. and Rubin, G.M. (1995) Gene Disruptions Using P Transposable Elements: An Integral Component of the Drosophila Genome Project. Proceedings of the National Academy of Sciences of the United States of America, 92, 10824-10830. http://dx.doi.org/10.1073/pnas.92.24.10824

[25] Timakov, B., Liu, X., Turgut, I. and Zhang, P. (2002) Timing and Targeting of P-Element Local Transposition in the Male Germline Cells of Drosophila melanogaster. Genetics, 160, 1011-1022.

[26] Sudi, J., Zhang, S., Intrieri, G., Hao, X.M. and Zhang, P. (2008) Coincidence of P-Insertion Sites and Breakpoints of Deletions Induced by Activating P Elements in Drosophila. Genetics, 179, 227-235. http://dx.doi.org/10.1534/genetics.107.085498

[27] Freeman, M. (1997) Personal Communication to FlyBase. http://flybase.org/reports/FBrf0091569.html

[28] Steffan, J.S., Agrawal, N., Pallos, J., Rockabrand, E., Trotman, L.C., Slepko, N., Illes, K., Lukacsovich, T., Zhu, Y.Z., Cattaneo, E., et al. (2004) SUMO Modification of Huntingtin and Huntington's Disease Pathology. Science, 304, 100104. http://dx.doi.org/10.1126/science.1092194

[29] Kazemi-Esfarjani, P. and Benzer, S. (2000) Genetic Suppression of Polyglutamine Toxicity in Drosophila. Science, 287, 1837-1840. http://dx.doi.org/10.1126/science.287.5459.1837

[30] Brand, A.H., Manoukian, A.S. and Perrimon, N. (1994) Ectopic Expression in Drosophila. Methods in Cell Biology, 44, 635-654.

[31] Yeh, P.-A., Yang, W.-H., Chiang, P.-Y., Wang, S.-C., Chang, M.-S. and Chang, C.-J. (2012) Drosophila Eyes Absent Is a Novel mRNA Target of the Tristetraprolin (TTP) Protein DTIS11. International Journal of Biological Sciences, 8 , 606-619. http://dx.doi.org/10.7150/ijbs.3782

[32] Duffy, J.B. (2002) GAL4 System in Drosophila: A Fly Geneticist’s Swiss Army Knife. Genesis, 34, 1-15. http://dx.doi.org/10.1002/gene.10150

[33] Fernandez-Funez, P., Nino-Rosales, M.L., de Gouyon, B., She, W.C., Luchak, J.M., Martinez, P., Turiegano, E., Benito, J., Capovilla, M., Skinner, P.J., et al. (2000) Identification of Genes That Modify Ataxin-1-Induced Neurodegeneration. Nature, 408, 101-106. http://dx.doi.org/10.1038/35040584

[34] Bilen, J. and Bonini, N.M. (2007) Genome-Wide Screen for Modifiers of Ataxin-3 Neurodegeneration in Drosophila. PLoS Genetics, 3, 1950-1964. http://dx.doi.org/10.1371/journal.pgen.0030177

[35] Zhang, S., Binari, R., Zhou, R. and Perrimon, N. (2010) A Genomewide RNA Interference Screen for Modifiers of Aggregates Formation by Mutant Huntingtin in Drosophila. Genetics, 184, 1165-1179. http://dx.doi.org/10.1534/genetics.109.112516

[36] Vossfeldt, H., Butzlaff, M., Prussing, K., Ni Charthaigh, R.A., Karsten, P., Lankes, A., Hamm, S., Simons, M., Adryan, B., Schulz, J.B., et al. (2012) Large-Scale Screen for Modifiers of Ataxin-3-Derived Polyglutamine-Induced Toxicity in Drosophila. PLoS ONE, 7, e47452. http://dx.doi.org/10.1371/journal.pone.0047452

[37] Moses, K. and Rubin, G.M. (1991) Glass Encodes a Site-Specific DNA-Binding Protein That Is Regulated in Response to Positional Signals in the Developing Drosophila Eye. Genes \& Development, 5, 583-593. http://dx.doi.org/10.1101/gad.5.4.583

[38] Ellis, M.C., O’Neill, E.M. and Rubin, G.M. (1993) Expression of Drosophila Glass Protein and Evidence for Negative Regulation of Its Activity in Non-Neuronal Cells by Another DNA-Binding Protein. Development, 119, 855-865.

[39] Mukhopadhyay, M. and Campos, A.R. (1995) The Larval Optic Nerve Is Required for the Development of an Identified Serotonergic Arborization in Drosophila melanogaster. Developmental Biology, 169, 629-643. http://dx.doi.org/10.1006/dbio.1995.1175

[40] de Velasco, B., Erclik, T., Shy, D., Sclafani, J., Lipshitz, H., McInnes, R. and Hartenstein, V. (2007) Specification and Development of the Pars Intercerebralis and Pars Lateralis, Neuroendocrine Command Centers in the Drosophila Brain. Developmental Biology, 302, 309-323. http://dx.doi.org/10.1016/j.ydbio.2006.09.035

[41] Moses, K., Ellis, M.C. and Rubin, G.M. (1989) The Glass Gene Encodes a Zinc-Finger Protein Required by Drosophila Photoreceptor Cells. Nature, 340, 531-536. http://dx.doi.org/10.1038/340531a0

[42] Rebay, I., Chen, F., Hsiao, F., Kolodziej, P.A., Kuang, B.H., Laverty, T., Suh, C., Voas, M., Williams, A. and Rubin, 
G.M. (2000) A Genetic Screen for Novel Components of the Ras/Mitogen-Activated Protein Kinase Signaling Pathway That Interact with the Yan Gene of Drosophila Identifies Split Ends, a New RNA Recognition Motif-Containing Protein. Genetics, 154, 695-712.

[43] Imarisio, S., Carmichael, J., Korolchuk, V., Chen, C.W., Saiki, S., Rose, C., Krishna, G., Davies, J.E., Ttofi, E., Underwood, B.R., et al. (2008) Huntington's Disease: From Pathology and Genetics to Potential Therapies. Biochemical Journal, 412, 191-209. http://dx.doi.org/10.1042/BJ20071619

[44] Poirier, M.A., Jiang, H.B. and Ross, C.A. (2005) A Structure-Based Analysis of Huntingtin Mutant Polyglutamine Aggregation and Toxicity: Evidence for a Compact Beta-Sheet Structure. Human Molecular Genetics, 14, 765-774. http://dx.doi.org/10.1093/hmg/ddi071

[45] Hartman IV, J.L., Garvik, B. and Hartwell, L. (2001) Principles for the Buffering of Genetic Variation. Science, 291, 1001-1004.

[46] Tong, A.H., Lesage, G., Bader, G.D., Ding, H.M., Xu, H., Xin, X.F., Young, J., Berriz, G.F., Brost, R.L., Chang, M., et al. (2004) Global Mapping of the Yeast Genetic Interaction Network. Science, 303, 808-813. http://dx.doi.org/10.1126/science.1091317

[47] Ooi, S.L., Pan, X.W., Peyser, B.D., Ye, P., Meluh, P.B., Yuan, D.S., Irizarry, R.A., Bader, J.S., Spencer, F.A. and Boeke, J.D. (2006) Global Synthetic-Lethality Analysis and Yeast Functional Profiling. Trends Genet, 22, 56-63. http://dx.doi.org/10.1016/j.tig.2005.11.003

[48] Ye, P., Peyser, B.D., Pan, X.W., Boeke, J.D., Spencer, F.A. and Bader, J.S. (2005) Gene Function Prediction from Congruent Synthetic Lethal Interactions in Yeast. MolSystBiol, 1, 0026. 